\title{
Blasting Vibration Generated by Breaking-Blasting Large Barriers with EBBLB
}

\author{
Wang Zhen-xiong, ${ }^{1}$ Gu Wen-bin, ${ }^{1}$ Liang Ting, ${ }^{2}$ Liu Jian-qing, ${ }^{1}$ Xu Jing-lin, ${ }^{1}$ and Liu Xin ${ }^{1}$ \\ ${ }^{1}$ College of Field Engineering, PLA University of Science and Technology, Nanjing, Jiangsu 210007, China \\ ${ }^{2}$ Graduate School, Xian International Studies University, Xian, Shaanxi 710128, China
}

Correspondence should be addressed to Gu Wen-bin; guwenbin1@aliyun.com

Received 31 March 2016; Revised 5 May 2016; Accepted 11 May 2016

Academic Editor: Tai Thai

Copyright ( 2016 Wang Zhen-xiong et al. This is an open access article distributed under the Creative Commons Attribution License, which permits unrestricted use, distribution, and reproduction in any medium, provided the original work is properly cited.

\begin{abstract}
Equipment for breaking and blasting large barriers (EBBLB) is new break-blast equipment, which inevitably induces ground vibration and may cause substantial damage to rock mass and nearby structures as well as human beings. The ground vibration induced by break-blast is one of the inevitable outcomes. By monitoring vibration at measuring points at different distances from blasting center, time history curve of vibrating velocity can be obtained; it can be drawn that blasting seismic waves are generated mainly from the explosion of the precharge. Furthermore, different approaches have been adopted to fit relationship between particle peak velocity (PPV) and distance from blasting center, comparative analysis of which provides the most appropriate relation expression to predict attenuation of PPV of vibration with distance from blasting center. The relation between vibration frequency and distance from blasting center is obtained by Fourier transform. And the research decomposes vibrating signals in vertical direction of different distances from blasting center with Hilbert-Huang Transform (HHT), extracting information of empirical mode components of blasting vibration signals; thus vibrating signals are contracted by spectrum information and energy information, three-dimensional energy, and energy attenuation of vibration with different distances from blasting center. The study can predict ground vibration generated by EBBLB and assess its damaging effects of blasting vibration for security and protection.
\end{abstract}

\section{Introduction}

Landslides caused by natural disasters such as earthquakes and debris flow may form a great number of large barriers on roads, bridges, and other transportation routes. Unless large barriers can be quickly cleared, rescue workers and large machinery cannot reach the disaster area in the fastest time, which hampers rescue progress seriously. Xu et al. proposed a barrier-breaking scheme with EBBLB [1]. Equipment in the scheme consists of two parts, the precharge of shaped charge and rear-charge with blasting bullets $[1,2]$. Precharge forms a hole in the obstacle by shaped effects and rear-charge enters barriers and explodes, which is more conducive for exploiting explosive energy smashing the boulder once and greatly reducing time for blasting barriers and emergency rescue [2].

Blasting vibration of EBBLB has a certain degree of damage on buildings and structures damaged by natural disasters; hence, it is necessary to study blasting vibration of new equipment. Damaging effects of blasting the boulder with EBBLB include blasting shock waves, seismic waves, and blasting flying rocks. Shock waves can be analyzed and studied by Air Bang theory [3]; blasting flying rocks are relatively near and can be easily controlled with a safety net; however, the study of seismic waves is the most complex. Different from the explosion of the large equivalent explosive in general rock soil medium, the total charge of the equipment is small and has different damaging effects. Consequently, its blasting vibration is much smaller than that of the large-scale explosion in general rock medium; thus, its damaging effect of blasting vibration is often overlooked. Several trials indicated that the ground tremors obviously for blasting vibration and stones slip down in the nearby districts, especially in geological disaster areas; barriers are usually located near the damaged buildings; therefore, even with vibrations smaller than damaging limitations, those 
cracks in buildings may extend [4]. Hence, when blasted by EBBLB, blasting vibration may cause secondary damage to the building. In addition to the geological conditions, the location of large boulder is more conducive to the spread of seismic waves; amplitude of its blasting vibration may be higher than that of the general soil mediums, so, to evaluate vibration damaging effects, it is imperative to study the blasting vibration of EBBLB (see Figure 1).

Main factors affecting blasting vibration are explosive performance, charging structure, geological conditions, and blasting distance [5]. Sambuelli proposed theoretical derivation of a peak particle velocity-distance law based on attenuation of seismic waves of charging amount and rock properties [6]; Blair showed blast vibration dependence on charge length, velocity of detonation, and layered media [7] and concluded that particle peak velocity (PPV) can be used as an indicator of the intensity of blasting vibration, seismic effects, and safety standards over a long period of repeated research and engineering practice. Seismic wave generated by EBBLB is an extremely complex wide-band nonstationary random wave, characterized with short time, abrupt change, and fast attenuation.

Yan and Baliktsis adopted neural network to predict attenuation law of blasting vibration $[8,9]$, but energy information carried by signals of blasting vibration cannot be profoundly analyzed; Hilbert-Huang signal processing put forward by Huang is considered as a breakthrough of linear and steady-state spectrum analysis based on Fourier transform and one of the newest time frequency local analysis methods recognized internationally [10-14]; Zhang and Li used HHT application for analysis of blasting vibration signals, finding that HHT can be effectively employed to extract information in blasting vibration signals [15]; Gong et al. analyzed similarities and differences between empirical mode decomposition and wavelet decomposition; characteristics of original signals can be better analyzed [16].

To study blasting vibration produced by blasting with originally developed EBBLB, PPV at different measuring points has been analyzed, demonstrating the relationship between PPV and distance from blasting center; HHT is employed to analyze vibration signals and get time frequency characteristics and distribution of vibration energy. Research results show attenuation law of seismic waves produced by EBBLB, effectively predicting its blasting vibration and contributing to preventing its damaging effects.

\section{Mechanism of Seismic Waves from EBBLB}

Mainly consisting of precharge and near charge in series, EBBLB has the design principles with ordinary series warheads of penetration blasting [17]. The precharge is shaped charge warhead and near-charge penetration blasting warhead. Precharge takes effect in the first place, utilizing shaped charge effect to form holes in rock and opening up path for near charge. Near-charge penetration blasting warhead enters rock along the path and continues penetration of kinetic energy. When the bullet enters the hole with a certain depth, the warhead explodes, smashing large barriers.

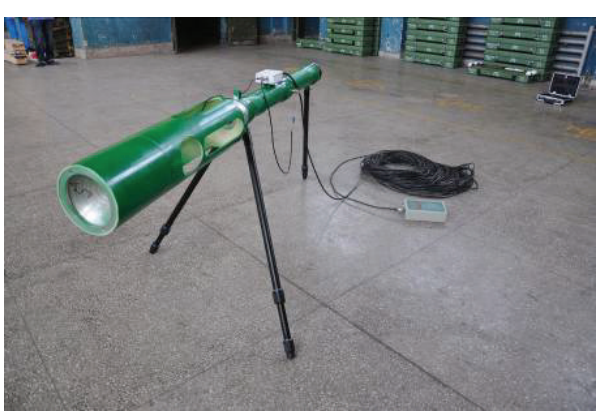

Figure 1: EBBLB.

When explosive explodes in the air, target from the explosion center is less than 15 times charging radius, within the scope of the joint action by explosive product and shock waves, and is greater than the distance, only by the air shock waves [18]. In the experiment, distance from charge to the ground is $1.4 \mathrm{~m}$ and charge radius is $0.14 \mathrm{~m}$, so the ground is acted upon by detonation products and air shock waves combined. Besides transmitting shock waves, explosion of precharge in the air also forms explosion craters of different degrees in rock surface. It also transmits shock waves to underground rock soil medium. After attenuating, shock waves form seismic waves in rock soil medium and transmit to the explosion far area.

Transmitting law of lasting vibration with EBBLB is different from that of ordinary blasting inside rocks. For the latter, explosion takes place inside rocks, generating explosion air with high temperature and high pressure that expands outward and shocks the rock; shock waves transmit inside the rock, rushing and fracturing rocks near the blasting center, while their wave velocity, pressure, and energy quickly attenuate with distance increasing; when they are far from the blasting center, shock waves inside the rock attenuate to a certain critical value and change into stress wave. Then, the inelastic process gradually ends, revealing elastic effect and continuing to transmit in the form of seismic waves [3].

In breaking and blasting with EBBLB, precharge explodes in the air while the rear-charge enters the blasting barrier and explodes inside it. There exists detonating delay between precharge and rear-charge, so it is more complicated than blasting vibration of ordinary rocks. In breaking and blasting boulder, precharge explodes in the air with a certain distance from the ground and there is no smashing area or crushing area on ground rocks, where the transmission of energy is different from that of ordinary blasting in rocks.

For the ground, the explosion belongs to the noncontact explosion, whose blasting seismic waves mainly come from two parts (shown in Figure 3): some come from the explosion of shaped charge in the air, forming stimulation to the ground and generating the seismic waves; some are generated because the explosion of traveling projectile in the boulder produces the elastic wave which transmits to the ground and forms the seismic waves. Ground seismic waves were generated by the air shock wave and elastic wave of the boulder, but as boulder shares relatively small contact area with the ground and they are not fixed together, elastic wave in the boulder 


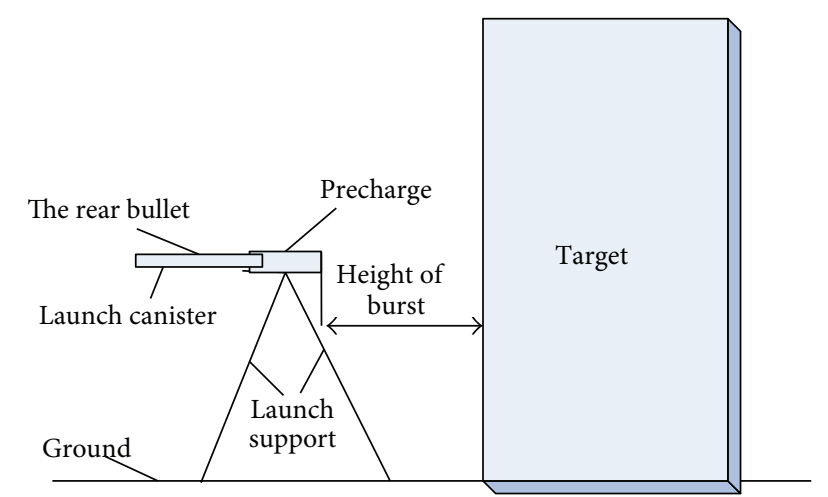

FIGURE 2: Schematic diagram of breaking and blasting large boulder with EBBLB.

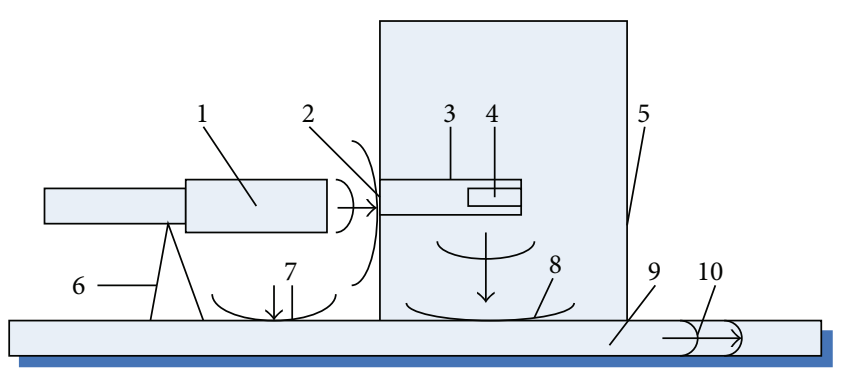

FIGURE 3: Schematic diagram of the formation of seismic waves. 1: shaped charge; 2: air shock wave and explosive detonation product; 3: penetration hole; 4: rear bullet; 5: boulder; 6: launch support; 7: air shock wave; 8: elastic wave; 9: ground; and 10: seismic waves.

reflects and transmits on the interface with the ground; then, energy transmitted to the ground decreases considerably; thus elastic wave transmitted from the boulder to the ground has relatively smaller influence on the seismic waves. Upon breaking and blasting with EBBLB (see Figure 2), the research measures the time history curves of vibration velocity that is similar to seismic waves form generated by the explosion in the air [6]; therefore, all that is needed for analysis is seismic waves generated by the explosion of precharge.

\section{The Signal Analysis Theory}

Hilbert-Huang Transform (HHT) consists of empirical mode decomposition (EMD) and Hilbert transform [10].

3.1. EMD Arithmetic. EMD is the key to HHT, and, in accordance with time scale of signals (time difference between adjacent peaks of signals), it decomposes signals to intrinsic mode function (IMF) which has different time scales and meets the following two conditions for the definition:

(a) In the entire data series, the number of extreme points and that of ZCP (Zero-Crossing Points) are equal to each other or differ by up to 1 .

(b) For any point on the signal, mean value of envelope line determined by local maximum value point and local minimum value point is 0 ; that is, the signal is locally symmetric about the time line.

EMD arithmetic is also called sifting process with the following calculation steps: first, find out all the extreme points on the original signal $x(t)$, interpolate all the extreme points by cubic spline function, and link all the maximum points to fit upper envelope of $x(t)$, and the same procedure may be easily adopted to obtain lower envelope. It should be assured that two envelops contain all the signal data. Then, define mean value of the two envelopes as $m_{1}$ and the difference of $x(t)$ and $m_{1}$ as

$$
h_{11}=x(t)-m_{1} \text {. }
$$

If $h_{11}$ fulfills the condition for defining, it is the first IMF, otherwise $h_{11}$ should be sifted repeatedly. Termination criterion of sifting process can be determined by SD of two continuous processed results:

$$
\mathrm{SD}=\sum_{t=0}^{T}\left|\frac{\left|h_{1(k-1)}(t)-h_{1 k}(t)\right|^{2}}{h_{1(k-1)}^{2}(t)}\right| .
$$

Generally, $h_{11}$ will not meet conditions for IMF. Supposing that, after sifting $k$ times, the result $h_{1 k}$ meets definition of IMF, then the first component of $x(t)$ is recorded as $c_{1}=h_{1 k}$. Then, make the difference of $x(t)$ and $c_{1} r(t)=x(t)-c_{1}(t)$ as the new signal data and repeat the above sifting process; components of IMF $c_{2}, c_{3}, \ldots, c_{n}$ can be obtained in turn. When the component $c_{n}$ or the margin $r_{n}$ is smaller than the present value or the margin $r_{n}$ becomes a monotonic function, the sifting process can be finished. With the above breakdown process, $x(t)$ can be decomposed as the sum of $n$ components of IMF and $r_{n}$ (see Figure 4):

$$
x(t)=\sum_{i=1}^{n} c_{i}(t)+r_{n}(t) .
$$

3.2. Hilbert Transform. After decomposing signals, many combinations of IMF can be obtained. Carry out Hilbert transform for each IMF component, instantaneous frequency of each IMF component can be obtained, and then Hilbert spectrum can be realized by synthesizing all the instantaneous frequencies of all the IMF components. Carry out Hilbert transformation on IMF component $c(t)$ of signal $x(t)$ :

$$
H[c(t)]=\frac{1}{\pi} \mathrm{PV} \int_{-\infty}^{+\infty} \frac{c\left(t^{\prime}\right)}{t-t^{\prime}} d t^{\prime} .
$$

Here, PV is Cauchy principal value. Then, signal $z(t)$ can be analyzed based on this structure:

$$
z(t)=c(t)+j(H)[c(t)]=a(t) e^{j \Phi(t)} .
$$

Here, $a(t)$ is amplitude value function and $\Phi(t)$ is phase function:

$$
\begin{aligned}
a(t) & =\sqrt{c^{2}(t)+H^{2}[c(t)]}, \\
\Phi(t) & =\arctan \frac{H[c(t)]}{c(t)} .
\end{aligned}
$$




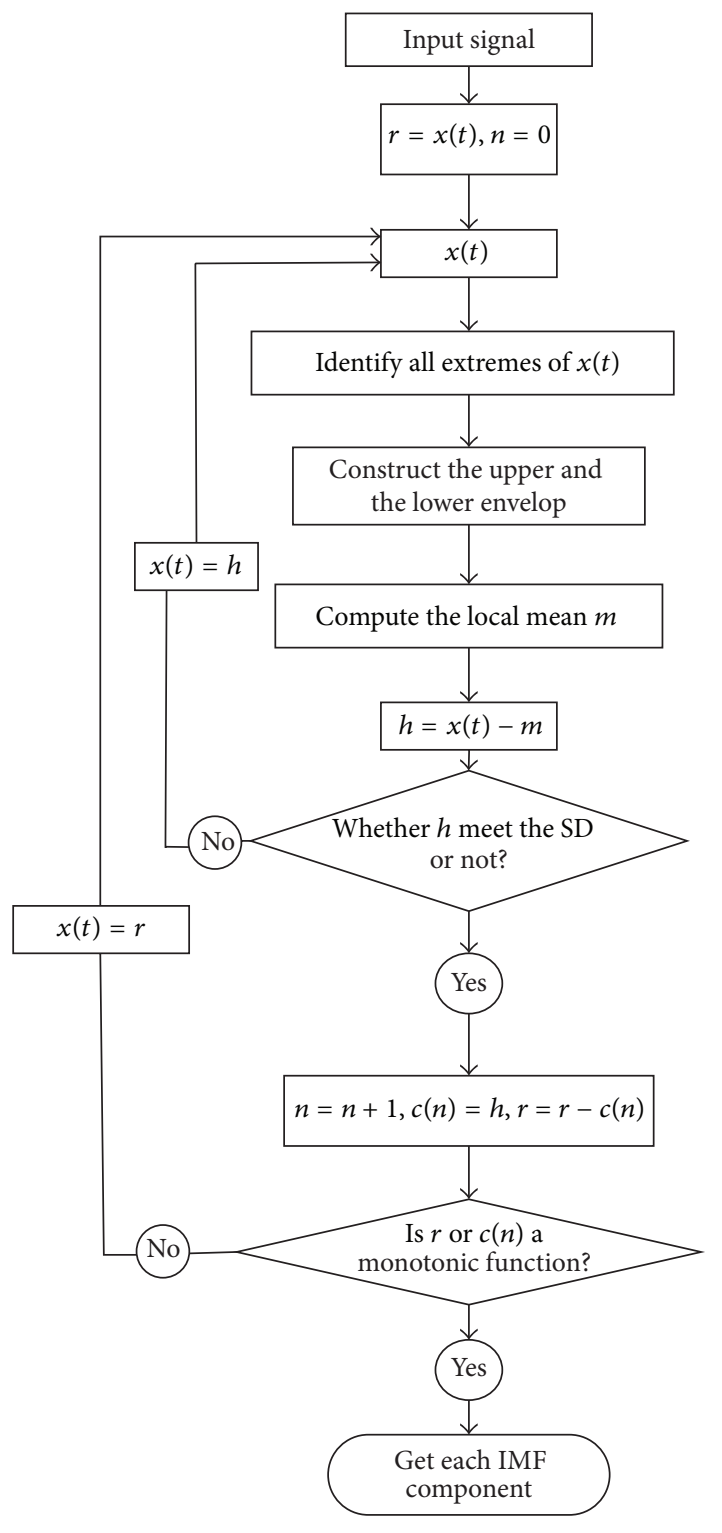

FIgURE 4: Calculation procedure diagram of EMD.

The angle-instantaneous frequency $\omega(t)$ and instantaneous frequency $f(t)$ obtained are

$$
\begin{aligned}
& \omega(t)=\frac{d \Phi(t)}{d t}, \\
& f(t)=\frac{d \Phi(t)}{2 \pi * d t} .
\end{aligned}
$$

For each component of the IMF after the Hilbert transform, the Hilbert spectrum expression is

$$
H(\omega, t)=\operatorname{Re} \sum_{i=1}^{n} a_{i}(t) e^{j_{\Phi_{i}(t)}}
$$

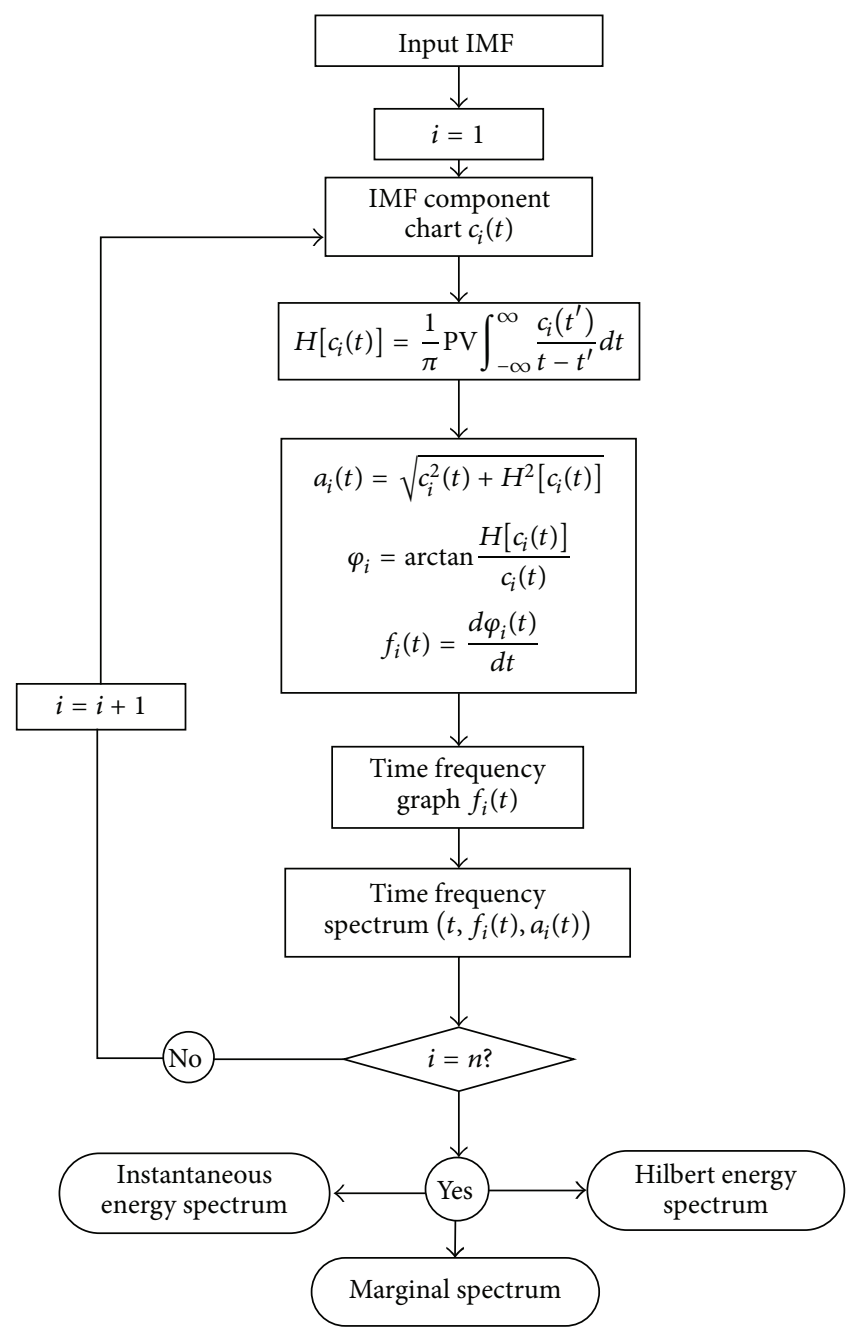

FIgURE 5: Calculation procedure diagram of Hilbert transform.

Hilbert marginal spectrum can be obtained from the time integration of $H(\omega, t)$ :

$$
h(\omega)=\int_{0}^{T} H(\omega, t) d t
$$

Hilbert instantaneous energy can be defined as

$$
\operatorname{IE}(t)=\int_{\omega} H^{2}(\omega, t) d t
$$

Instantaneous energy shows how signal energy changes over time. And Hilbert energy spectrum can be obtained from the time integration of squared amplitude:

$$
\mathrm{ES}(\omega)=\int_{0}^{T} H^{2}(\omega, t) d t
$$

Hilbert energy offers calculation formula of energy for each frequency, revealing energy accumulated of each frequency in the whole time duration (see Figure 5). 


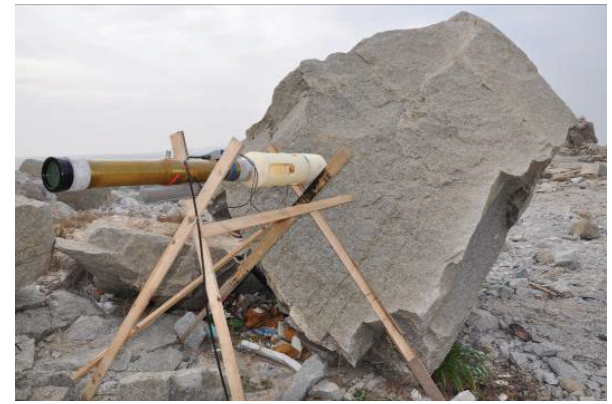

FIGURE 6: Setup diagram of blasting experiment with breaking and blasting equipment.

3.3. Advantage of HHT Method. HHT is characterized by easy application, adaptability, and high efficiency. The method breaks the traditional limitation of defining harmonic signals with unchanged amplitude as basement and it is unnecessary to preselect primary function; therefore, original signal can be decomposed into a few IMF components of actual physical significance, arranged from higher frequency to lower frequency, which is convenient for extracting and analyzing signal characteristics.

Instantaneous frequency obtained through Hilbert conversion has clear physical significance and is able to show local features of the signal, showing change relationships between energy and time as well as frequency accurately, which is more appropriate for processing nonstationary signal compared to Fourier analysis method and wavelet analysis algorithm that rely on transcendental function basis. Thus, HHT method is more appropriate for signal analysis of breaking and blasting vibration that is featured by quick mutation and quick attenuation.

\section{Experimental Condition and Test System}

To avoid damage effects of breaking and blasting boulder with EBBLB, the experiment (see Figure 6) is set at Dazhui Island where there are more boulders and no buildings around. Dazhui Island is located at the mouth of Quanzhou Bay in Huian County, Quanzhou city in Fujian province, $2.3 \mathrm{~km}$ from the coast. Main ingredient of rocks on the island is sedimentary rock whose weathering degree is not high and mass integrity is good. Originally developed EBBLB with detonation delay in order has been adopted to blast boulder and breaking and blasting equipment is set as is showed in Figure 3. Shaped charged structure designed by $\mathrm{Xu}$ et al. has been selected as precharge. Charge mass is $4.8 \mathrm{~kg}$; aluminized explosives of $0.48 \mathrm{~kg}$ have been selected as rear-charge. Launch canister is improved by DZP11-80 air explosive bomb launch canister with individual distance $[1,2]$.

Testing system of vibration employed TC-4850 tester (see Figure 7) of blasting vibration, which can record vibration signals in horizontal, vertical, and radial directions. Sampling frequency is $1 \sim 50 \mathrm{KHz}$ and frequency range is $0 \sim 10 \mathrm{KHz}$, with better stability and higher reliability. Based on conditions of

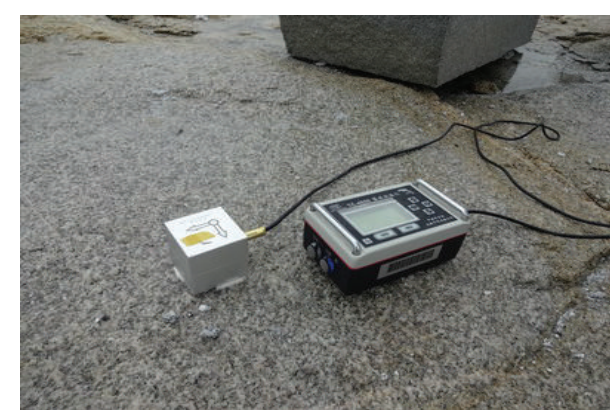

FIGURE 7: Setup diagram of vibration tester TC-4850.

TABLE 1: Blasting vibration data $(\mathrm{cm} / \mathrm{s})$.

\begin{tabular}{lllll}
\hline$R(\mathrm{~m})$ & $v_{x-\max }$ & $v_{y \text {-max }}$ & $v_{z-\max }$ & $\mathrm{PPV}$ \\
\hline 20 & 1.398 & 1.062 & 1.555 & 2.345 \\
50 & 0.598 & 0.286 & 1.049 & 1.241 \\
80 & 0.665 & 0.185 & 0.315 & 0.759 \\
120 & 0.125 & 0.069 & 0.035 & 0.147 \\
160 & 0.032 & 0.047 & 0.119 & 0.132 \\
\hline
\end{tabular}

test site, measuring points in a line are selected, and the sensor is fixed on a smooth rock by cast, with $X$ direction of sensor aimed at blasting center and $Z$ direction perpendicular to rock surface. To ensure the cast bonding sensor and rock are entirely coagulated, the sensor should be set 10 minutes before carrying out the blasting.

\section{Analysis of Vibration Signals}

5.1. Analysis of Vibration Velocity. According to conditions of test site, vibration data of measuring points within the scope of $20 \mathrm{~m}-160 \mathrm{~m}$ from blasting center are collected. Figure 8 is time history curve of vibration velocity of measuring points that are $20 \mathrm{~m}$ and $160 \mathrm{~m}$ from blasting center (see Figure 9). Peak values of vibration velocity of measuring points are shown in Table 1.

Table 1 indicates that peak values of vibration velocity in three directions are different and the maximum value may appear in any direction; hence, resultant velocity should be adopted for analysis, which can synthetically show vibration velocity in three directions and prevent the impact of direction errors in setting measuring points [19].

It is reasonable to choose square root from the square sum of three portions' peak speed as the particle's peak vibration velocity. The value of peak vibration velocity of the particle is [20]

$$
\operatorname{PPV}=\left|v_{\text {sum }}\right|=\sqrt{\left(v_{x-\max }\right)^{2}+\left(v_{y-\max }\right)^{2}+\left(v_{z-\max }\right)^{2}} .
$$

Analysis on formation mechanism of seismic waves of blasting boulder with EBBLB indicates that, in fitting formula of vibration velocity, the impact of explosive payload of rear bullet can be ignored and explosive payload of precharge is regarded as a single explosive dosage for analysis and calculation (see Figure 10). 

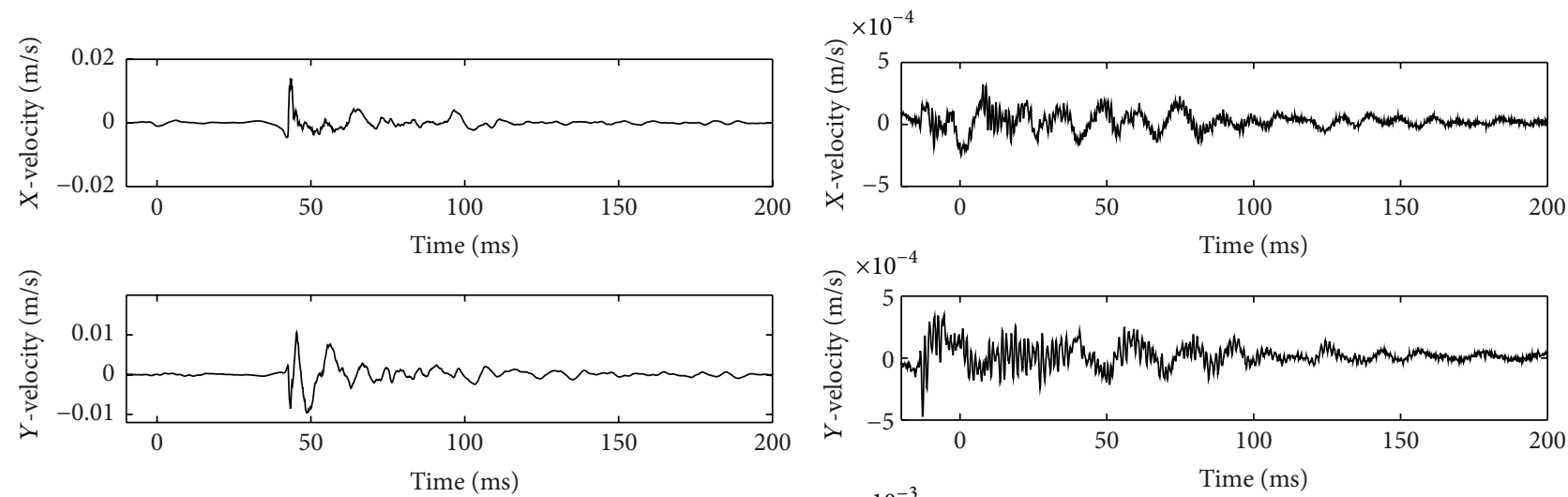

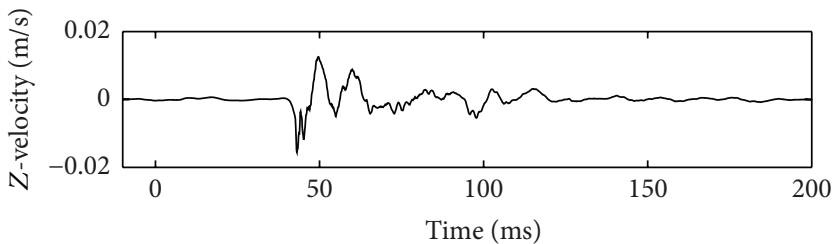

(a)

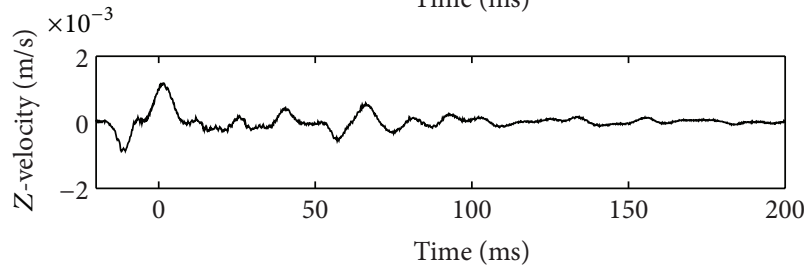

(b)

FIGURE 8: Time history curve of vibration velocity at measuring points with $20 \mathrm{~m}$ (a) and $160 \mathrm{~m}$ (b) from blasting center.

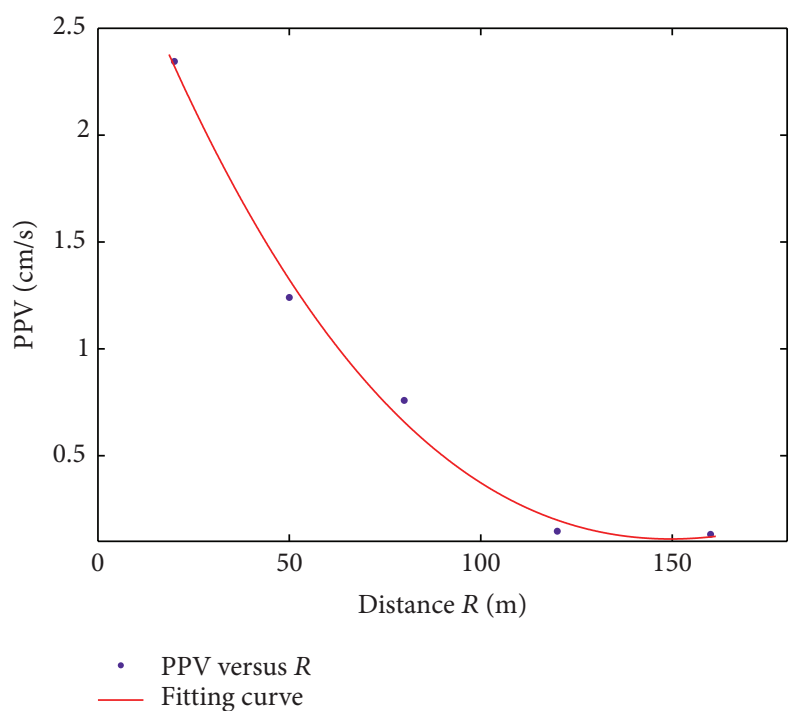

FIGURE 9: Changing curve of vibration velocity with distance from blasting center.

Based on geometrical similarity principle, a relation between peak value of vibration velocity of the particle peak velocity (PPV), charging mass $(Q)$, and distance from blasting center $(R)$ can be obtained [21]:

$$
\mathrm{PPV}=k\left(\frac{R}{\sqrt[3]{Q}}\right)^{-\alpha}=k r^{-\alpha}
$$

In the formula, $k$ is coefficient related to explosion condition and vibration transmission; $\alpha$ is index number of attenuation; and $r$ is scaled distance. After disposing data in

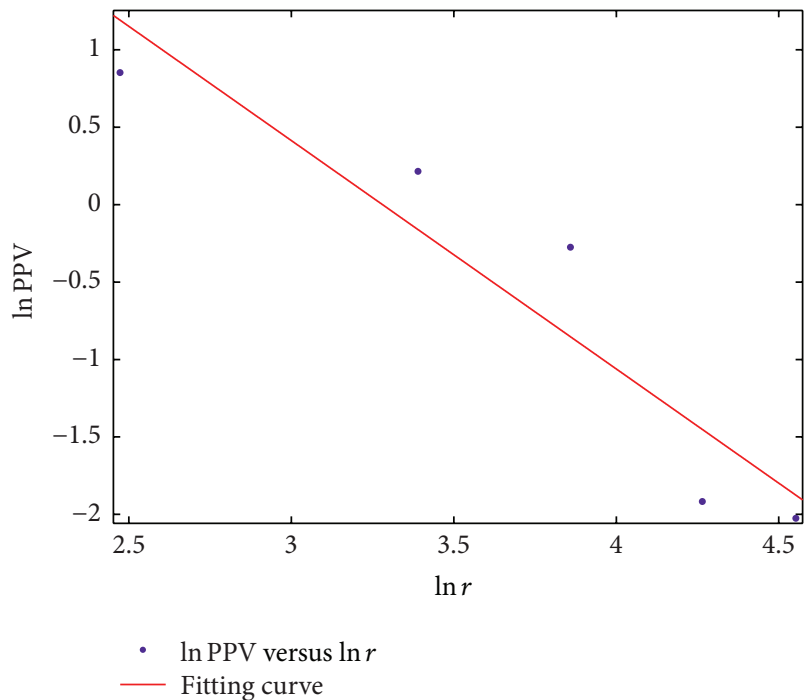

Figure 10: Curve of fitted data.

Table 1, change law of vibration velocity with distance from blasting center can be fitted by least square method:

$$
\mathrm{PPV}=126.217 r^{-1.475} \quad(20 \leq R \leq 160)
$$

Correlation coefficient in the fitting formula (14) is just 0.936 and mean square error is 0.525 . Calculation of vibration velocity of different distances from blasting center reveals that great error exists between calculated value and experimental value, as Table 1. Figure 9 shows vibration velocity with the increase of distance from blasting center. Polynomial is 
employed to fit the change of vibration velocity with distance from blasting center $(20 \mathrm{~m}-160 \mathrm{~m})$ :

$$
\begin{aligned}
\mathrm{PPV}= & 3.086-0.042 \times R+1.474 \times 10^{-4} \times R^{2}, \\
\mathrm{PPV}= & 3.196-0.048 \times R+2.303 \times 10^{-4} \times R^{2} \\
& -3.089 \times 10^{-7} \times R^{3} .
\end{aligned}
$$

Correlation coefficient of binomial fitting (formula (15)) is 0.993 and mean square error is 0.107 . Correlation coefficient of trinomial fitting (formula (16)) is 0.994 and $s$ mean square error is 0.143 . Comparisons of calculated value and experimental value of the above three fitting formulas are shown in Table 2.

Through fitting of vibration data by three formulas, it is known that data fitted by Sadove formula has greater error and its prediction on vibration velocity generated by EBBLB is inaccurate. Fitted result by trinomial has smaller error, which is closer to experimental value. Optimization selection of prediction formula of vibration velocity proves that formula (16) can better predict blasting vibration velocity generated by blasting with EBBLB [22].

When the new blasting equipment EBBLB is applied to area of natural disaster, seismic waves generated in blasting barrier may damage surrounding buildings; hence, it is necessary to study its blasting vibration. Seismic wave generated by blasting with EBBLB is different from ordinary rock blasting in intensity and transmission law. Analysis of transmission law of seismic waves by PPV reveals that intensity of seismic waves exponentially attenuates with distance from blasting center. Greater error exists in formula fitted by ordinary prediction of rock vibration, while calculation formula fitted by polynomial has higher correlation coefficient and smaller standard deviation that can satisfy requirements to predict peak value of vibration velocity.

According to safe standard on blasting vibration in GB6722-2003, with reference to allowable vibration velocity for such protection objects as loess cave dwelling, row houses, and stone houses, allowable safety vibration velocity is $0.5 \sim$ $1.5 \mathrm{~cm} / \mathrm{s}$ [23].

Calculation with fitting formula (16) indicates that when distance from blasting center is $59.093 \mathrm{~m}$, vibration velocity is $1.1 \mathrm{~cm} / \mathrm{s}$; when the distance is $78.371 \mathrm{~m}$, vibration velocity is $0.7 \mathrm{~cm} / \mathrm{s}$; and vibration velocity is $0.5 \mathrm{~cm} / \mathrm{s}$ with the distance of $91.170 \mathrm{~m}$, suggesting that if distance from blasting center is larger than $100 \mathrm{~m}$, damage caused by blasting vibration can be ignored. Thus, when EBBLB is employed, to avoid damaging effects on buildings, the safety distance should be $100 \mathrm{~m}$.

5.2. Analysis on Vibration Frequency. As an important factor influencing damaging effects of blasting vibration, vibration frequency should be paid attention to. Blasting vibration velocity in Section 5.1 reveals that vibration velocity generated by blasting with distance from blasting center of $100 \mathrm{~m}$ is beyond allowable safety vibration velocity for such protection objects as loess cave dwelling, row houses, and stone houses in GB6722-2003 [23]; therefore, the research analyzes vibration frequency at measuring points $20 \mathrm{~m}, 50 \mathrm{~m}$, and $80 \mathrm{~m}$ from the blasting center. Through the Discrete Fourier Transform (DFT), power spectrum of three measuring points in different directions can be obtained [24, 25] (as depicted in Figure 11).

In signal processing, frequency corresponding to spectral maximum in power spectrum is usually called dominant frequency and it can be adopted to analyze frequency spectrum characteristics of vibration under different blasting conditions in engineering. Figure 11 shows that measuring point with smaller distance from blasting center has higher dominant frequency of $93.75 \mathrm{~Hz}$; dominant frequencies at measuring points of $50 \mathrm{~m}$ and $80 \mathrm{~m}$ are closer, that is, $19.53 \mathrm{~Hz}$ and $17.58 \mathrm{~Hz}$, which are far smaller than that of measuring point of $20 \mathrm{~m}$. Thus, when distance from blasting center is smaller, the vibration amplitude is larger and dominant frequency is relatively higher, while, with the increase of distance from blasting center, vibration velocity index attenuates, and dominant frequency also decreases dramatically.

5.3. HHT Analysis on Vibration Signals. Fourier transform can relate time-domain characteristics in blasting signals to frequency-domain characteristics and analyze signal characteristics from time-domain and frequency-domain, respectively. But it fails to combine the two effectively and lacks complete time-domain information, while HHT decomposes vibrational signals according to time-scale characteristics of the data [15]. To further study information of seismic waves generated by blasting and breaking boulders, vibration signals at measuring points of $20 \mathrm{~m}$ and $50 \mathrm{~m}$ in vertical directions are selected and analyzed by HHT method (shown in Figure 12).

The original signals can be decomposed by EMD method and components are shown in Figure 13. Original signals are decomposed as 13 IMF components including $c_{1}-c_{12}$ and an allowance. According to the time scale from large to small, high frequency is decomposed first and low frequency second. Most IMF components decomposed have clear physical significance. Among them, $c_{1}$ has the highest frequency and the shortest wave length. With the decomposition, frequency of IMF components gradually gets lower; wave length gets longer, till it decomposes an allowance with very low frequency. Component $c_{1}$ has the highest frequency and contains very small energy which is well distributed in signals, suggesting it is high-frequency noise brought in monitoring and needs denoising in further analysis; $c_{2}$ and $c_{3}$ are high frequency in vibration signals consisting of small energy because high frequency sharply attenuates in seismic waves transmission. Components $c_{4}, c_{5}, c_{6}$, and $c_{7}$ have lower frequency and increased amplitude. They are dominant frequency band, containing most energy of signals. Thus, damage to structures mainly comes from these components, which should be focused on. Besides, IMF after $c_{8}$ is decomposed component with smaller frequency, which may be inherent in signals or caused by other situations. Final allowance indicates zero drift of monitoring instrument or variation tendency of weak signals.

Figure 13 reveals that, in EMD decomposing results with distance from blasting center of $20 \mathrm{~m}$, component $c_{4}$ has the largest amplitude and $c_{5}$ has the largest amplitude with 
TABLE 2: Comparison of calculated value and experimental value of three fitting formulas $(\mathrm{cm} / \mathrm{s})$.

\begin{tabular}{lcccccc}
\hline$R$ & \multicolumn{2}{c}{ Formula (14) } & \multicolumn{2}{c}{ Formula (15) } & \multicolumn{2}{c}{ Formula (16) } \\
& Calculated value & Error (\%) & Calculated value & Error (\%) & Calculated value & Error (\%) \\
\hline $20 \mathrm{~m}$ & 3.289 & 40.26 & 2.305 & 1.71 & 9.326 & 1.333 \\
$50 \mathrm{~m}$ & 0.851 & 31.43 & 1.355 & 11.86 & 0.672 & 7.41 \\
$80 \mathrm{~m}$ & 0.426 & 43.88 & 0.669 & 14.29 & 0.218 & 11.46 \\
$120 \mathrm{~m}$ & 0.234 & 59.18 & 0.168 & 21.97 & 0.135 & 2.38 \\
$160 \mathrm{~m}$ & 0.153 & 15.91 & 0.103 & & \\
\hline
\end{tabular}
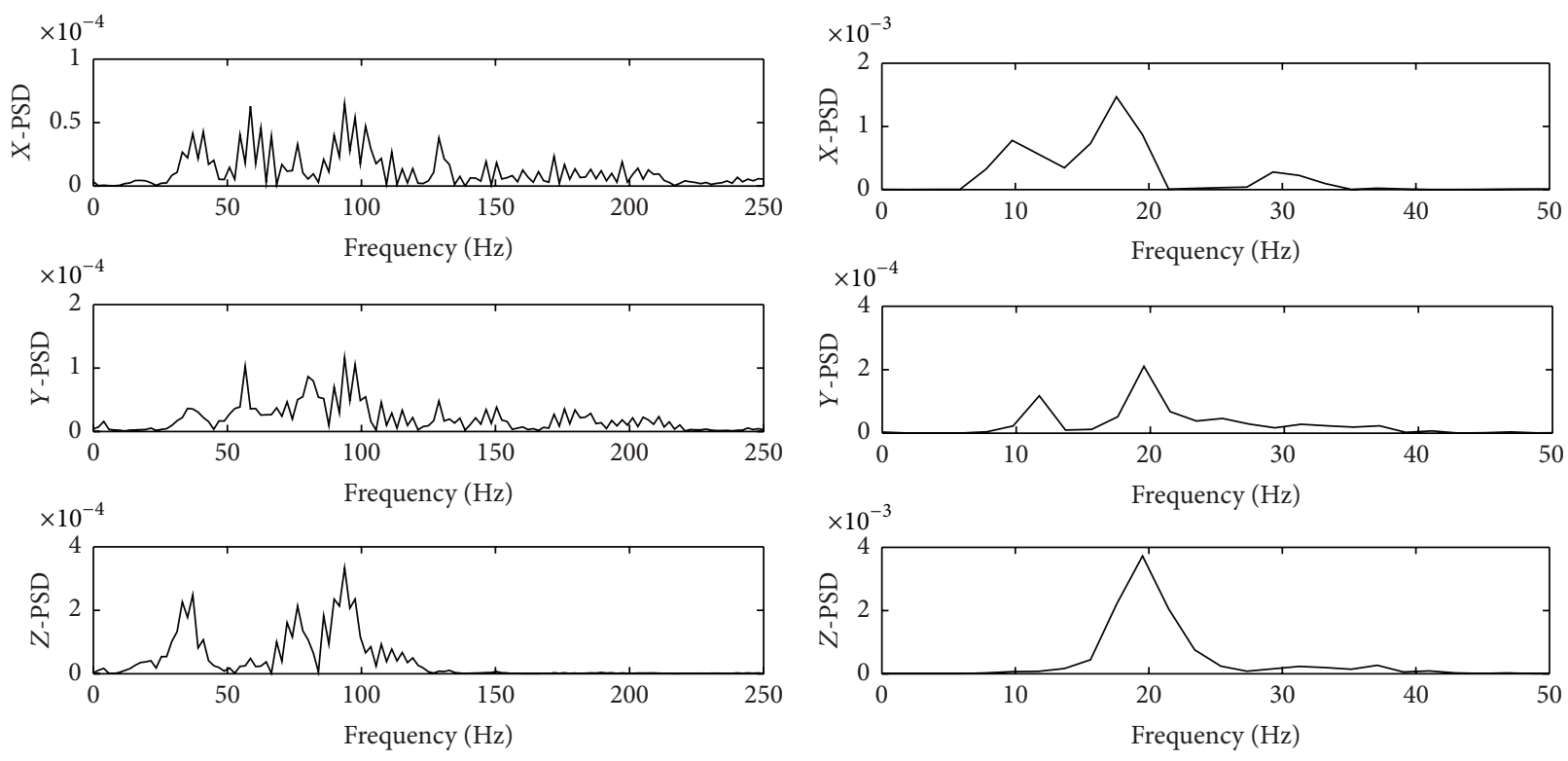

(a) $20 \mathrm{~m}$

(b) $50 \mathrm{~m}$
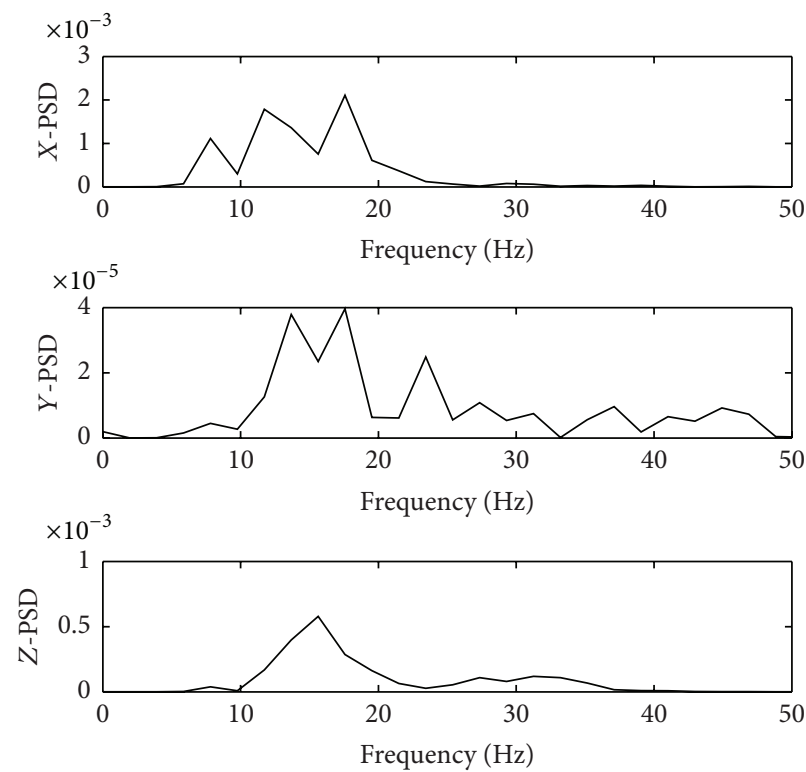

(c) $80 \mathrm{~m}$

Figure 11: Power spectrum chart of vibration signals at different measuring points in three directions. 

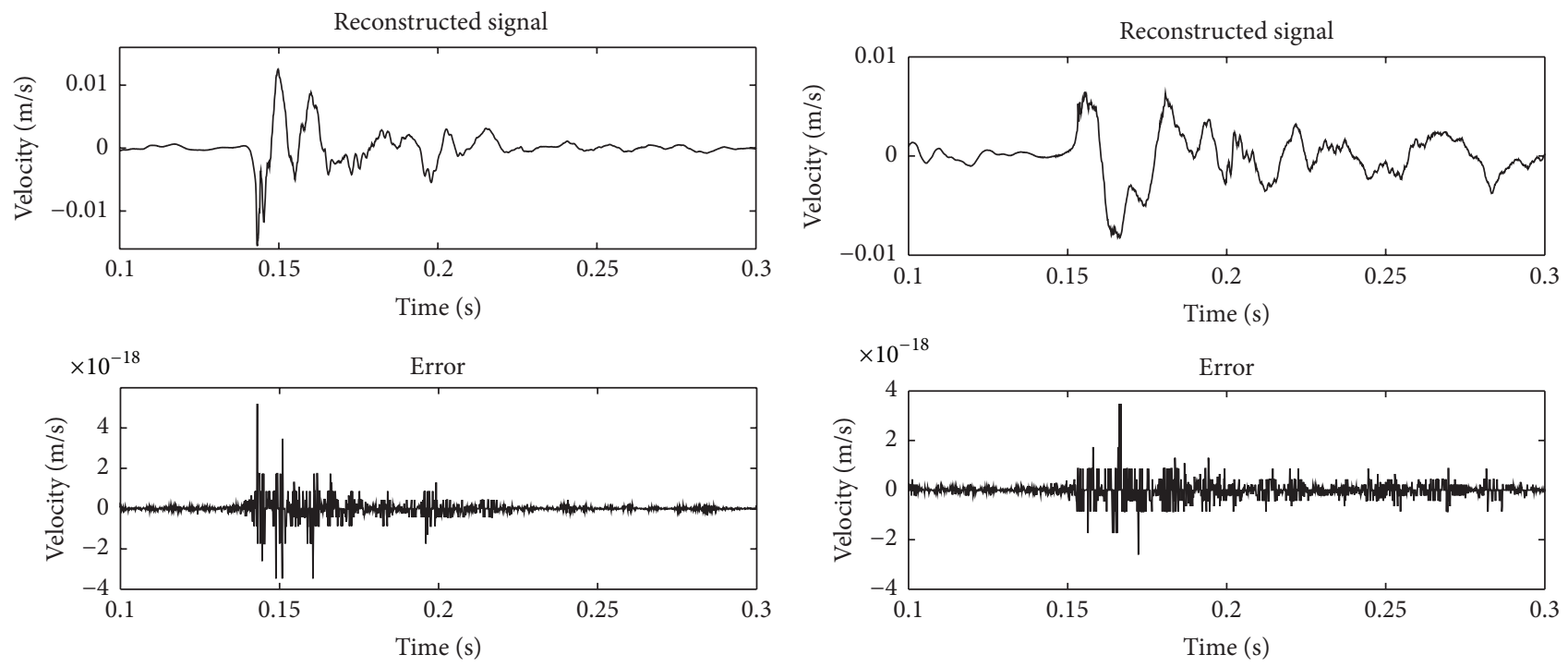

(a) $20 \mathrm{~m}$

(b) $50 \mathrm{~m}$

FIGURE 12: Time history curve and reconstructing signals and error graph of blasting vibration velocity at different measuring points.

distance of $50 \mathrm{~m}$, showing that, with increase of distance from blasting center, signals' dominant frequency decreases. Components $c_{4}, c_{5}, c_{6}$, and $c_{7}$ contain most energy of the signal and they are the main reason for damaging structures and buildings. There is little error between original signals and reconstituted signals by IMF components. Figure 12 indicates the error level is $10^{-18}$, which can completely satisfy requirements of engineering calculation and analysis and verify reliability of EMD decomposition.

EMD decomposition has adaptability on change of signals; hence, IMF components decomposed from vibration signals at measuring points with different distances from blasting center are different; spectral distributions of IMF components vary a lot. With analysis on frequency spectrum of IMF components $c_{4}-c_{7}$ (shown in Figure 14), dominant frequencies of components $c_{4}-c_{7}$ at measuring point of $20 \mathrm{~m}$ are 93.75, 72.27, 37.11, and 25.39; dominant frequencies of components $c_{4}-c_{7}$ at measuring point of $50 \mathrm{~m}$ are $33.20,17.58$, 11.72, and 9.77. Basic frequency of component $c_{4}$ of vibration signals at measuring point of $20 \mathrm{~m}$ from blasting center and that of component $c_{5}$ at measuring point of $50 \mathrm{~m}$ are in accordance with dominant frequency of Section 5.2, revealing that components decomposed by EMD retain main information of original signals. Comparison of two spectrograms in the figure indicates that frequency of vibration signals decreases with the increase of distance from blasting center and, especially for components containing main information, frequency attenuates more apparently.

Instantaneous energy graph of original signals can be obtained through HHT method (shown in Figure 15). The figures clearly reflect how energy of vibration signals changes with time change. Comparison on instantaneous energy graph at measuring points with different distances from blasting center shows that instantaneous energy at measuring point with $20 \mathrm{~m}$ to blasting center has higher amplitude, the distribution is more concentrative, and duration is shorter. And amplitude of the instantaneous energy at measuring point $50 \mathrm{~m}$ from blasting center decreased, while distribution range of vibration signals is bigger and the vibration duration increases.

Figure 16 is three-dimensional energy spectrum of vibration signals at different measuring points in vertical direction, reflecting distribution of blasting vibration energy over time and frequency. Comparing the three-dimensional energy spectrums, researchers find that blasting vibration energy at measuring points near the blasting center is more dispersed, among which high-frequency component contains more energy and shorter duration, while, at measuring point $50 \mathrm{~m}$ from blasting center, the energy is mainly distributed in lowfrequency part, with longer duration. Different from instantaneous energy amplitude in Figure 16, energy amplitude of measuring point of $20 \mathrm{~m}$ is smaller than that of $50 \mathrm{~m}$.

\section{Discussions}

EBBLB is new blasting and breaking equipment. When it is applied to natural disaster areas, seismic waves of blasting may damage surrounding buildings, so it is necessary to study its blasting vibration. Different from blasting in general rock blasting, seismic wave generated by blasting with EBBLB is different in both strength and dissemination law. After analyzing dissemination law of seismic waves by PPV, it is found that seismic waves' strength exponentially attenuates with change of distance from blasting center, and there exists obvious error in formula fitted with predicting method of vibration in general rocks. Nevertheless, formula obtained by polynomial fitting has relatively higher correlation coefficient and smaller standard deviation and can meet requirements of predicting peak value of vibration velocity. With reference to GB6722-2003, safety permitted standard of blasting 

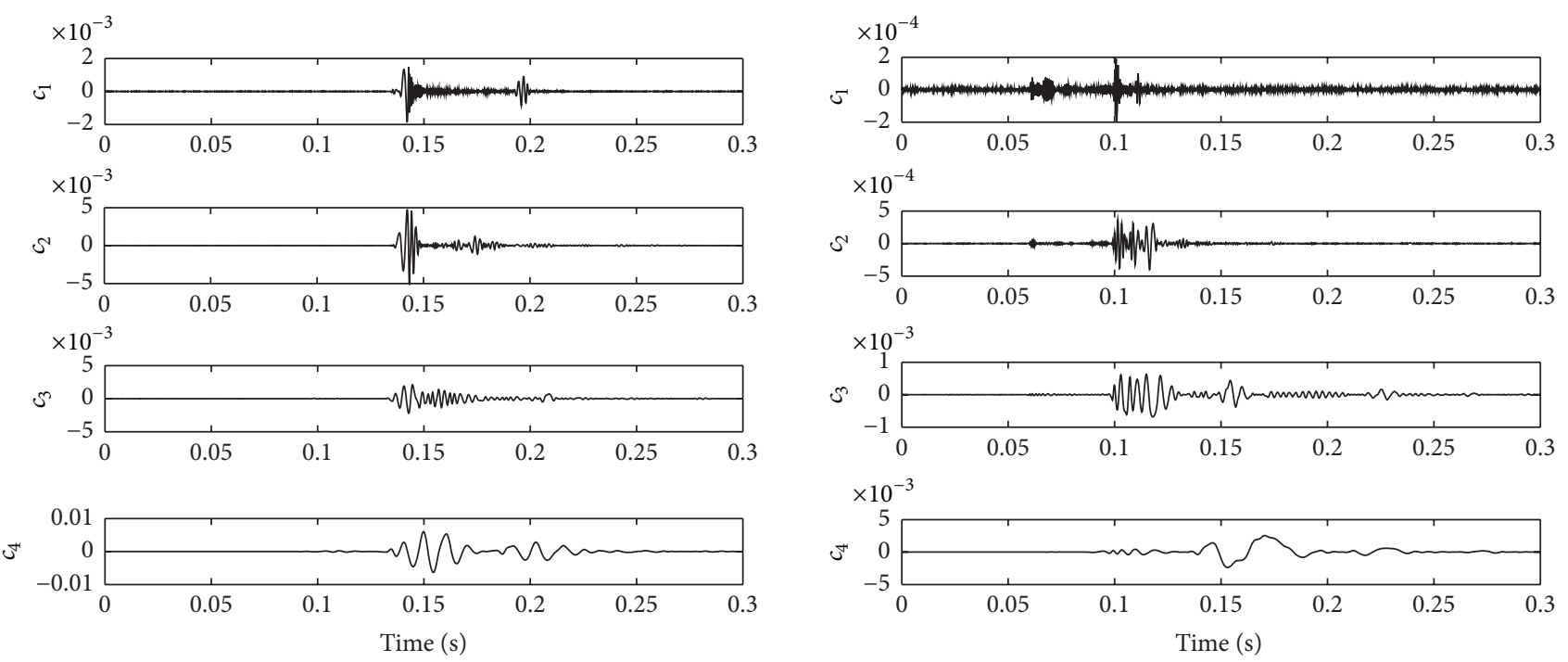

(1)

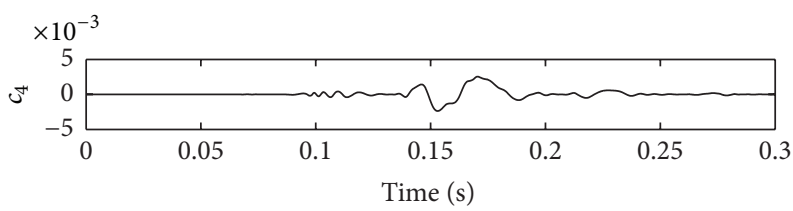

(1)
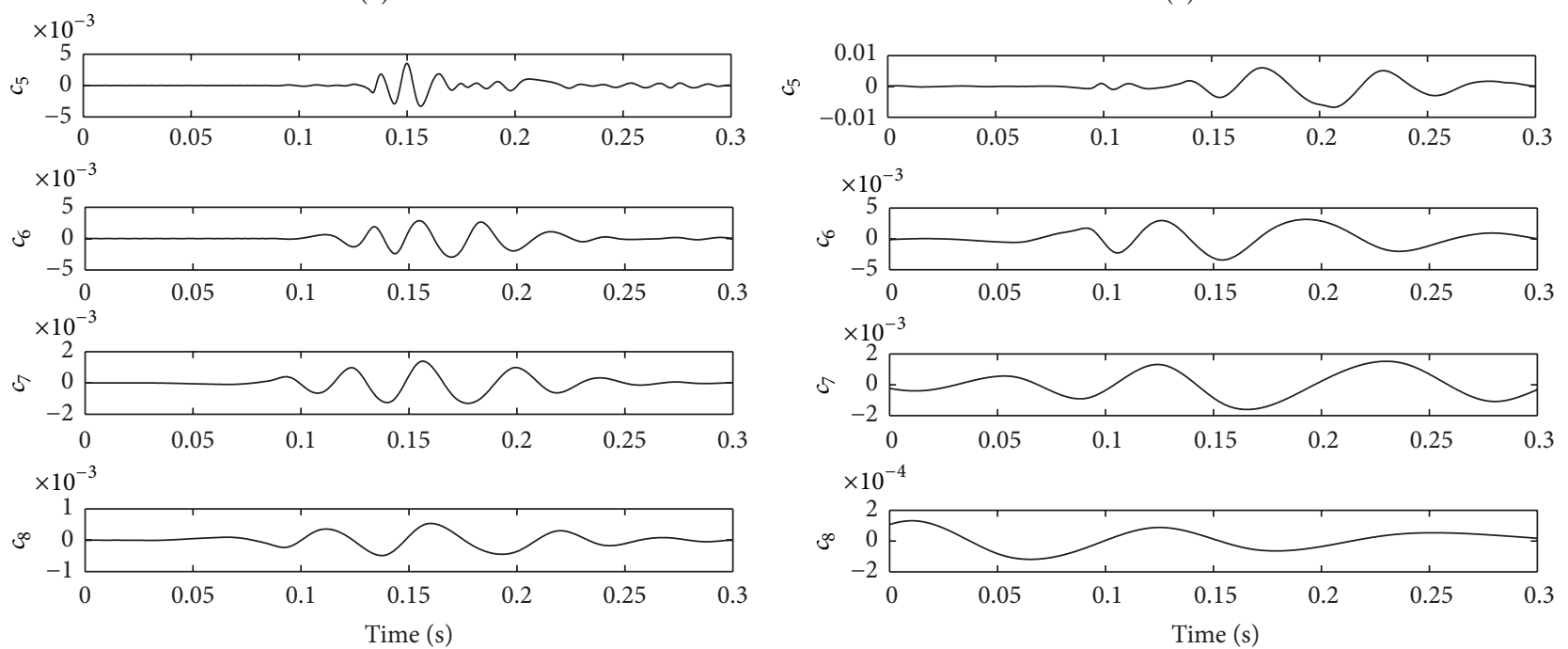

(2)
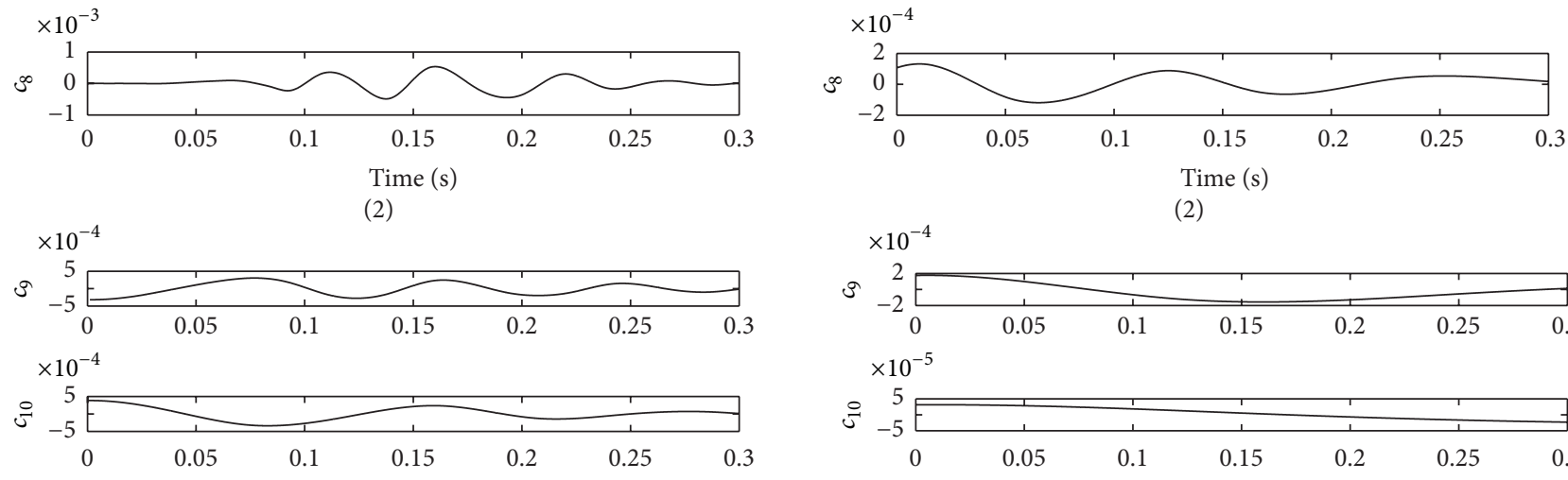

(2)
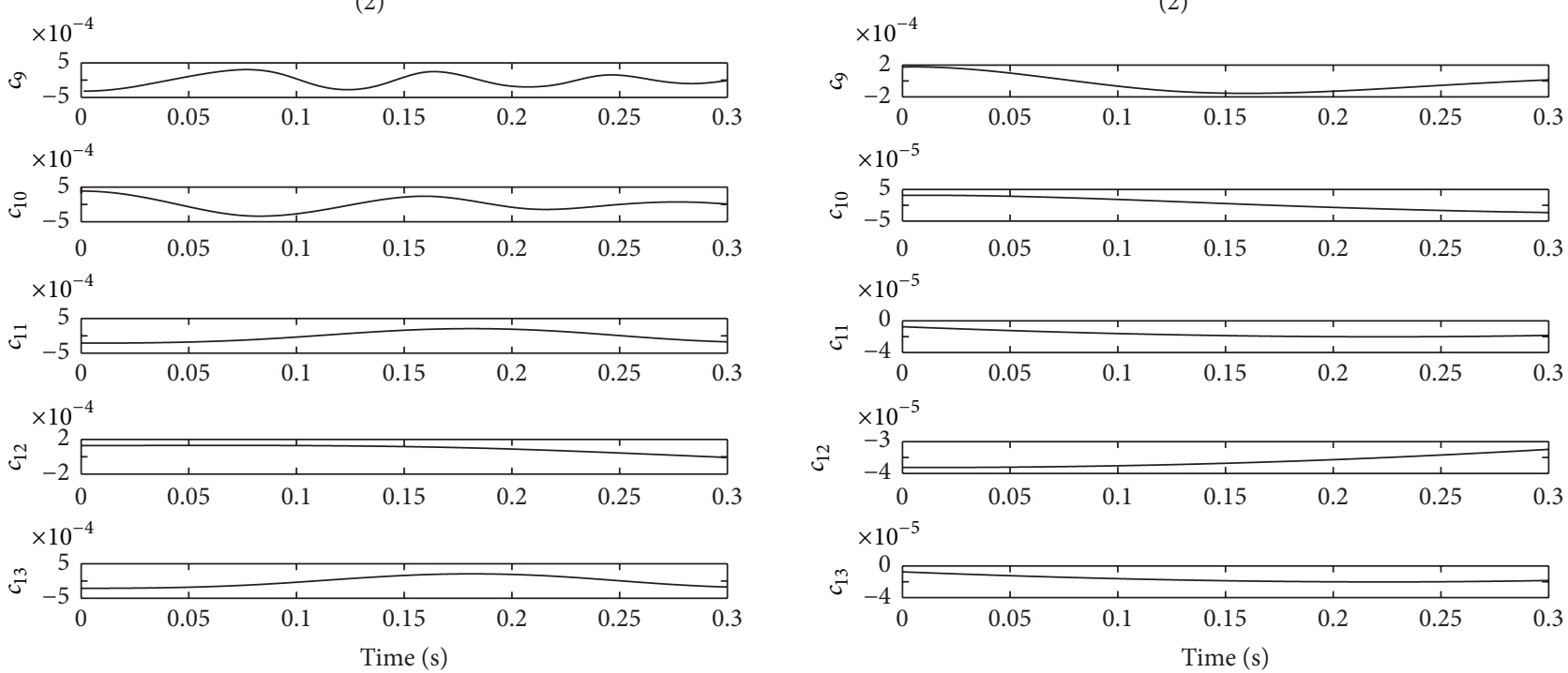

(3)

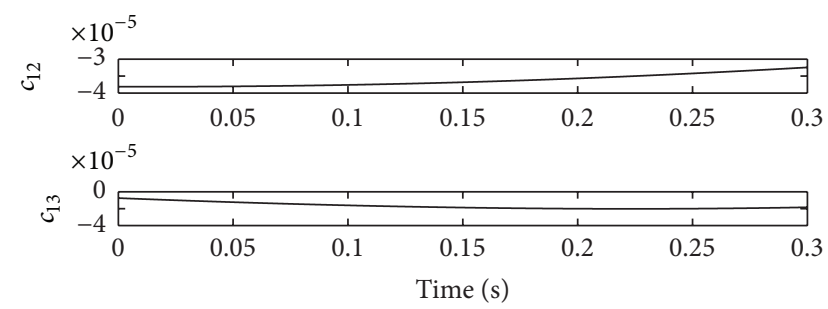

(3)

(a) $20 \mathrm{~m}$

(b) $50 \mathrm{~m}$

FIGURE 13: EMD decomposing results of blasting vibration signals at different measuring points. 

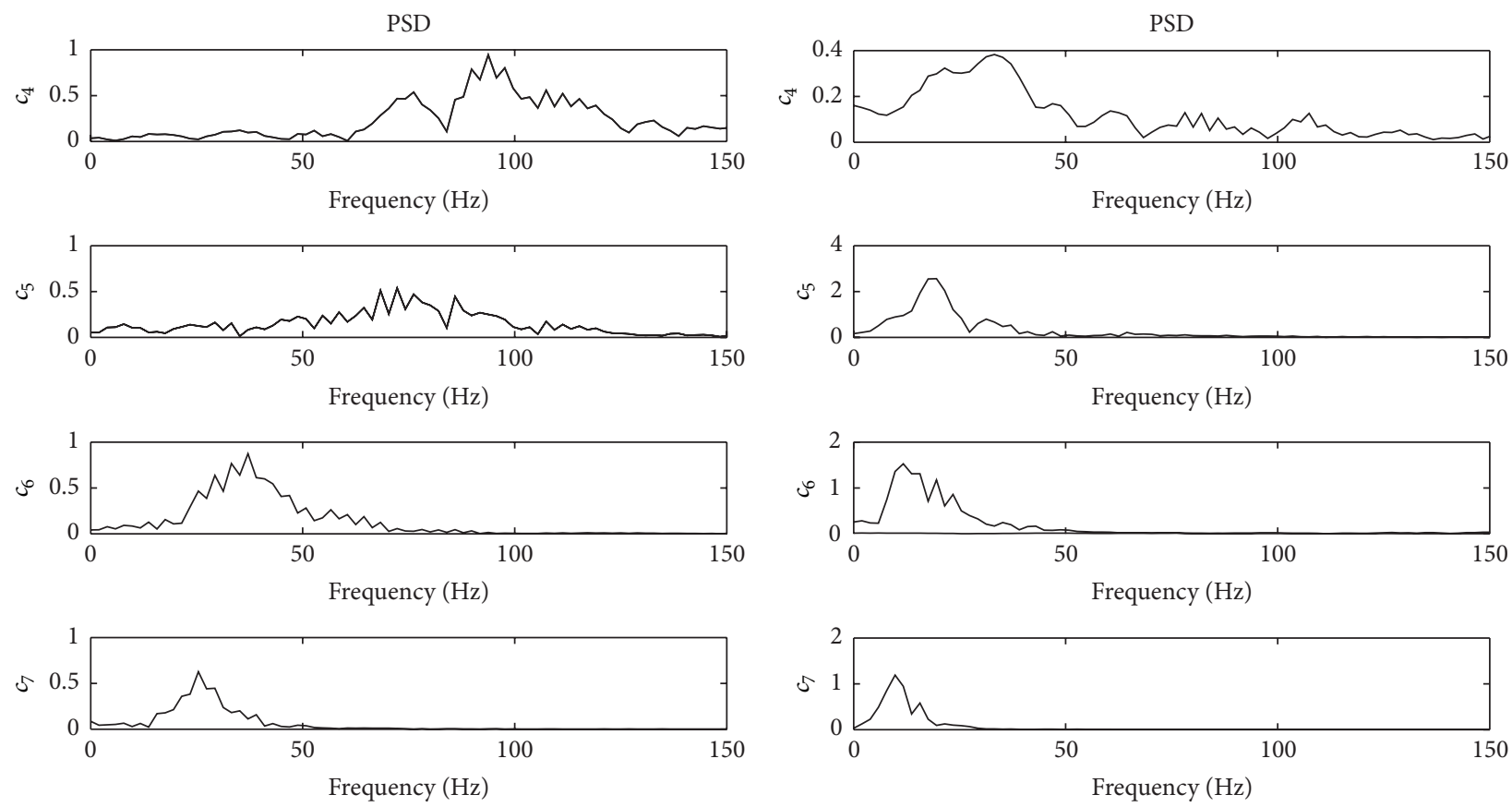

(a) $20 \mathrm{~m}$

(b) $50 \mathrm{~m}$

FIGURE 14: Spectrogram of components $c_{4} \sim \mathcal{c}_{7}$ of EMD decomposition at different measuring points.

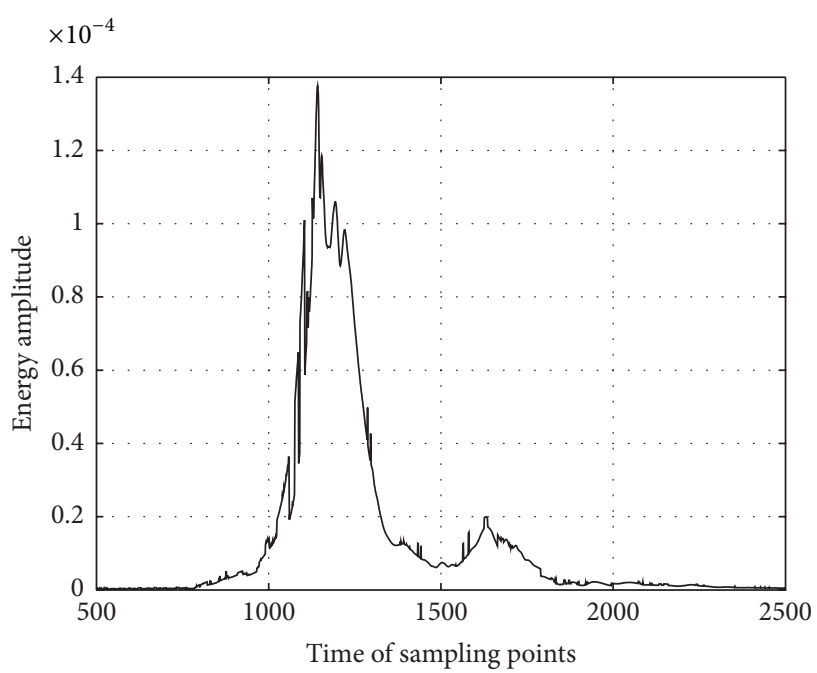

(a) $20 \mathrm{~m}$

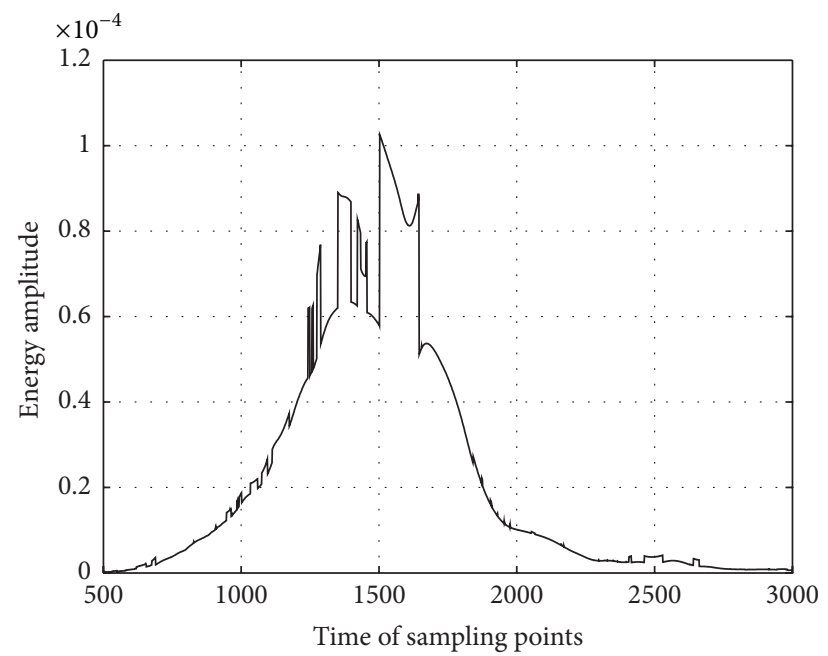

(b) $50 \mathrm{~m}$

FIGURE 15: Instantaneous energy graph of vibration signals at different measuring points.

vibration suggests that application of EBBLB should ensure safety distance of $100 \mathrm{~m}$ for protecting buildings. Analysis on blasting vibration signals through Fourier Change shows that, with the increase of distance from blasting center, frequency of seismic waves decreases obviously, because seismic waves are mainly made up of four components, that is, longitudinal wave (P-wave), transverse wave ( $\mathrm{S}$-wave), Rayleigh wave (Rwave), and Love waves (L-waves) [26], wherein longitudinal and transverse waves belong to body waves and Rayleigh and Love waves belong to surface waves. Different waves have various transmission velocity and frequency. Body waves compress and stretch transmission medium, have high frequency, and mainly act in near zone of blasting, while surface waves have low frequency, large amplitude, and slow attenuation, carrying more energy and spreading wider. So, with increase of blasting distance, amplitude and frequency 

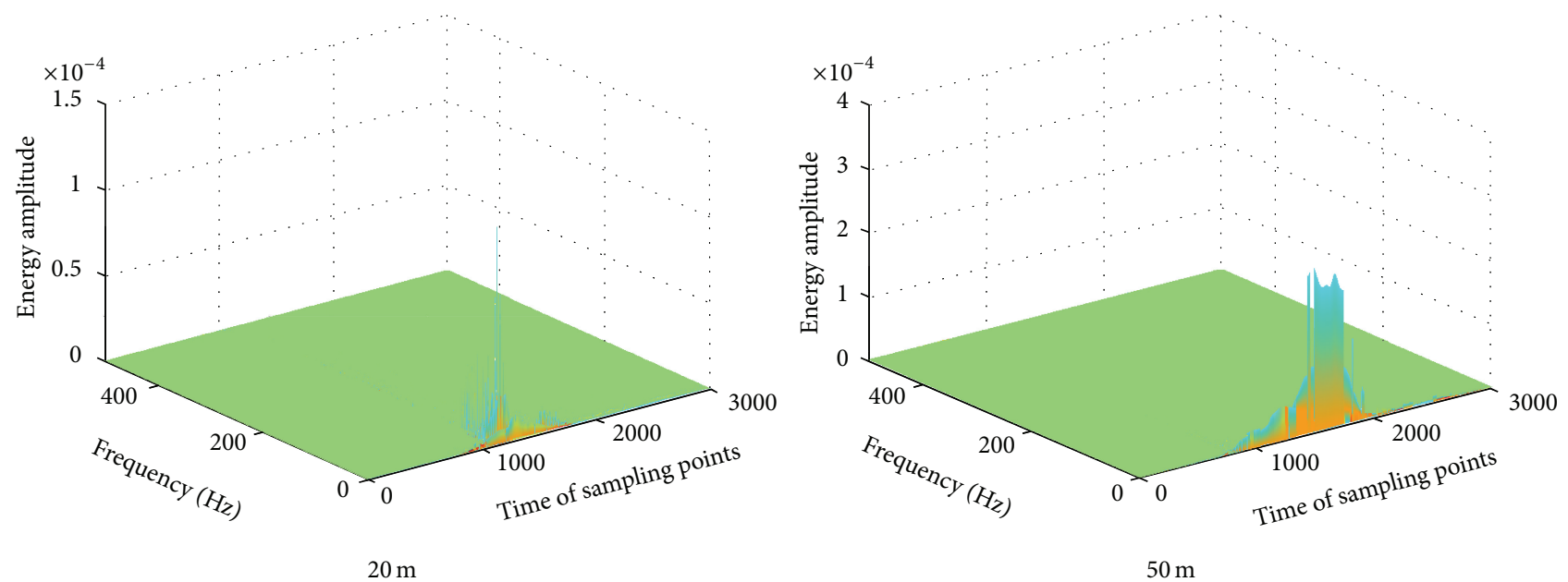

FIGURE 16: Three-dimensional energy spectrum of vibration signals at different measuring points.

of blasting seismic waves attenuate greatly, but, meanwhile, various waves gradually separate, with duration of blasting vibration extending.

HHT can effectively extract main characteristics of signals changes and adapt to analysis on blasting vibration signals featured by quick mutation and quick attenuation. Magnitude order of the error between signals decomposed and restructured by HHT method and the original ones is $10^{-16}$, which can meet requirements of analysis. With HHT method, different vibration signals can be decomposed from high to low frequency; then components having larger influence can be extracted. In this way, frequency spectrum of different components can be obtained, together with instantaneous energy and three-dimensional energy graph of vibration signals at different time. Comparing and analyzing IMF components of vibration signals at measuring points of $20 \mathrm{~m}$ and $50 \mathrm{~m}$ in vertical directions by HHT method, researchers find that amplitude of component $c_{4}$ with distance from blasting center of $20 \mathrm{~m}$ is the largest. And, in components of $50 \mathrm{~m}, c_{5}$ component of lower frequency has the largest amplitude. With the increase of distance from blasting center, main frequency of signals decreases. Comparison of four IMF components that carry main energy apparently reveals distribution of signal frequency. Instantaneous energy reflects vibration energy carried by vibration signals at different time. When distance from blasting center increases, maximum of instantaneous energy decreases, but amplitude obviously gets wider. With increase of distance, various waves in seismic waves gradually separate and duration of blasting vibration is prolonged. Hilbert three-dimensional energy spectrum clearly shows distribution of energy over time frequency. Most energy is concentrated on limited energy spectra line. When transmission distance increases, total energy carried by vibration signals constantly attenuates and high-frequency components attenuate faster than lowfrequency components. Energy proportion of intermediateand low-frequency signal components increases, with main frequency of blasting vibration signals getting lower. As natural vibration frequency of structure buildings is relatively low, in transmission of seismic waves, though vibration strength constantly attenuates, its damaging effects may increase.

\section{Conclusions}

Through studying seismic waves produced by blasting boulder with EBBLB, the following conclusions can be drawn:

(1) Rear-charge can be ignored in studying total charge quantity produced by blasting with EBBLB. Only the mass of precharge should be considered in fitting formula of vibration velocity.

(2) Different from transmission law of seismic waves generated by general rock blasting, polynomial fitting correlation is better for expressing relationship between particle peak velocity (PPV) generated by blasting boulders with EBBLB and distance from blasting center; that is, $\mathrm{PPV}=3.196-0.0048 \times R+$ $2.303 \times 10^{-4} \times R^{2}-3.089 \times 10^{-7} \times R^{3}$, and safety allowable distance of blasting vibration with EBBLB is $100 \mathrm{~m}$.

(3) With Fourier transform in vibration signals at different measuring points, results show that when distance from blasting center increases, index of vibration velocity attenuates, and dominant frequency greatly decreases.

(4) IMF components decomposed by EMD have certain physical meaning, and there exists minimum error between reconstructed signal and original signal. Comparison and analysis of vibration signals of $20 \mathrm{~m}$ and $50 \mathrm{~m}$ in vertical direction show that frequency of blasting vibration signals as well as instantaneous energy amplitude of near measuring points is relatively high; with increase of distance, signal frequency and instantaneous energy obviously decrease, but duration of blasting vibration increases. Threedimensional energy spectrum indicates that, for far distance, vibration energy is mainly of low-frequency components, which are main energy on damaging 
structure and building; therefore, damaging effect of near distance is not necessarily larger than that of far distance.

\section{Competing Interests}

The authors declare that they have no competing interests.

\section{Acknowledgments}

This work was supported by grants from Department of Infrastructure Barracks and National Science-Technology Support Plan, Grants nos. BY209J033 and 2012BAK05B01. Their contribution to bringing this project to fruition is much appreciated.

\section{References}

[1] H.-M. Xu, W.-B. Gu, and H. Huang, "Numerical simulation and experimental investigation on a shaped charge with new structure liner," in Proceedings of the International Conference on Mechanical Design, Manufacture and Automation Engineering, Phuket, Thailand, January 2014 (Chinese).

[2] H.-M. Xu, W.-B. Gu, Z. Zeng, Z.-X. Wang, and C.-X. Zhao, "Effect of delay time on formation and penetration of tandem EFP," Chinese Journal of High Pressure Physics, vol. 28, no. 1, pp. 79-85, 2014 (Chinese).

[3] L. P. Orlenko, Explosion Physics, Science Press, Beijing, China, 2011.

[4] P. K. Singh and M. P. Roy, "Damage to surface structures due to blast vibration," International Journal of Rock Mechanics \& Mining Sciences, vol. 47, no. 6, pp. 949-961, 2010.

[5] D.-C. Lin, Q. Zhang, and C.-H. Bai, "Random property of ground vertical vibration velocity induced by near ground explosion seismic effect," Expolosion and Shock Waves, vol. 20, no. 3, pp. 235-240, 2000 (Chinese).

[6] L. Sambuelli, "Theoretical derivation of a peak particle velocitydistance law for the prediction of vibrations from blasting," Rock Mechanics and Rock Engineering, vol. 42, no. 3, pp. 547-556, 2009.

[7] D. P. Blair, "Blast vibration dependence on charge length, velocity of detonation and layered media," International Journal of Rock Mechanics and Mining Sciences, vol. 65, pp. 29-39, 2014.

[8] Z.-X. Yan and H.-Y. Wang, Blasting Vibration Effect and Safety, Science Press, Beijing, China, 2001 (Chinese).

[9] E. K. Baliktsis, D. C. Kaliampakos, and D. G. Damigos, "Blasting vibration limits to prevent human annoyance remarks from some case studies," Mineral Resources Engineering, vol. 10, no. 1, pp. 71-82, 2001.

[10] N. E. Huang, Z. Shen, and S. R. Long, "The empirical mode decomposition and the Hilbert spectrum for nonlinear and non-stationary time series analysis," Proceedings of Royal Society of London, vol. 454, no. 1971, pp. 903-995, 1998.

[11] K. Semion, T. P. Flatley, and N. E. Huang, On the Hilbert-Huang Transform Data Processing System Development, National Aeronautics and Space Administration Goddard Space Flight Center. Darrell Smith Orbital Science Corporation, 2001.

[12] S. K. Lee, Y. W. Kim, M. H. Koo, H. I. Gimm, and H. H. Yoo, "Hilbert-Huang Transform (HHT) transient analysis of composite panel undergoing high-velocity impact," Journal of
Mechanical Science and Technology, vol. 24, no. 12, pp. 23952400, 2010

[13] N. E. Huang and Z. Wu, "A review on Hilbert-Huang transform: method and its applications to geophysical studies," Reviews of Geophysics, vol. 46, no. 2, 23 pages, 2008.

[14] G. G. U. Aldas and B. Ecevitoglu, "Waveform analysis in mitigation of blast-induced vibrations," Journal of Applied Geophysics, vol. 66, no. 1-2, pp. 25-30, 2008.

[15] Y.-P. Zhang and X.-B. Li, "Application of Hilbert-Huang transform in blasting vibration signal analysis," Journal of Central South University, vol. 36, no. 5, pp. 882-887, 2005 (Chinese).

[16] Z.-Q. Gong, M.-W. Zou, X.-Q. Gao, and W.-J. Dong, "On the difference between empirical mode decomposition and wavelet decomposition in the nonlinear time series," Acta Physica Sinica, vol. 54, no. 8, pp. 3947-3957, 2005 (Chinese).

[17] S.-H. Wang and S.-S. Wang, "The research on efficiency assessment method of tandem penetrating blast warhead," Journal of Projectiles, Rockets, Missiles and Guidance, vol. 30, no. 5, pp. 121130, 2010 (Chinese).

[18] J.-G. Ning, C. Wang, and T.-B. Ma, Explosion and Shock Dynamics, National Defense Industry Press, Beijing, China, 2010, (Chinese).

[19] X.-Q. Xie, Precision Blasting, Huazhong University of Science and Technology Press, Wuhan, China, 2010, (Chinese).

[20] W.-B. Gu, Z.-X. Wang, J.-H. Chen, J.-Q. Liu, and M. Lu, "Experimental and theoretical study on influence of different charging structures on blasting vibration energy," Shock and Vibration, vol. 2015, Article ID 248739, 11 pages, 2015.

[21] C. H. Dowing, Blast Vibration Monitoring and Control, vol. 3, Prentice Hall, Englewood Cliffs, NJ, USA, 1985.

[22] W.-G. Bi and C. Shi, "Optimization selection of blasting vibration velocity attenuation formulas," Rock and Soil Mechanics, vol. 25, supplement, pp. 99-102, 2004 (Chinese).

[23] X.-G. Wang, T.-R. Xu, Z.-Y. Zhang, Z.-Q. Wang, and D.-Z. Liu, GB6722-2003 Safety Regulation for Blasting, China Standard Press, Beijing, China, 2004 (Chinese).

[24] D.-F. Zhang, MATLAB Numerical Analysis, China Machine Press, Beijing, China, 2012 (Chinese).

[25] X.-B. Li, T.-H. Ling, and Y.-P. Zhang, Analysis of Blasting Vibration Signals Theories and Methods, Science Press, Beijing, China, 2009 (Chinese).

[26] S. D. Butt, D. B. Apel, and P. N. Calder, "Analysis of high frequency microseismicity recorded at an underground hardrock mine," Pure and Applied Geophysics, vol. 150, no. 3-4, pp. 693704, 1997. 


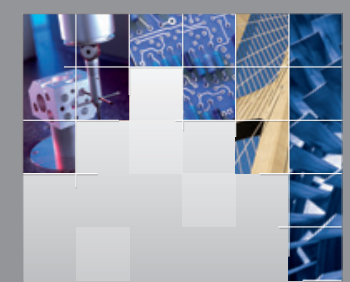

\section{Enfincering}
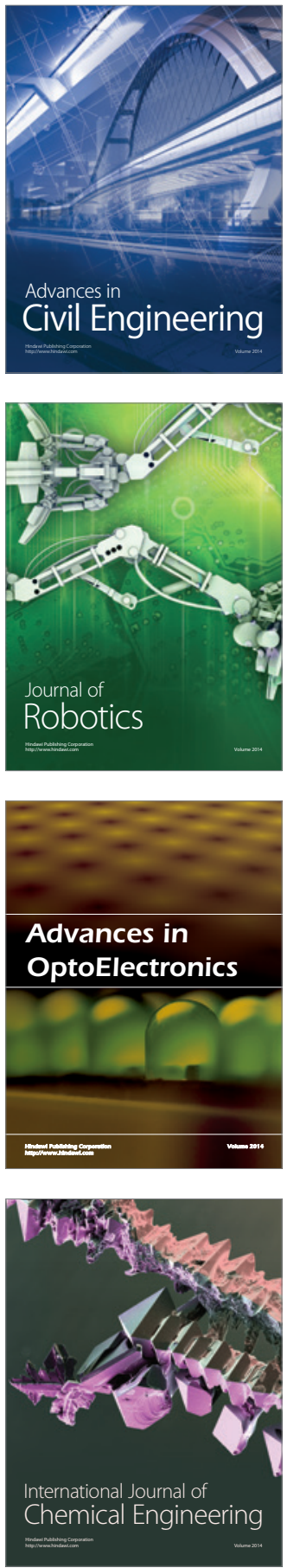

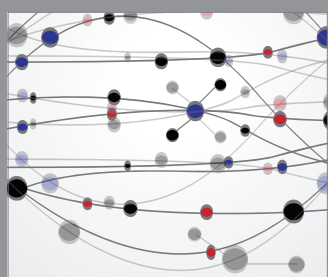

The Scientific World Journal

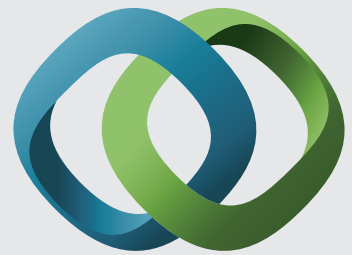

\section{Hindawi}

Submit your manuscripts at

http://www.hindawi.com
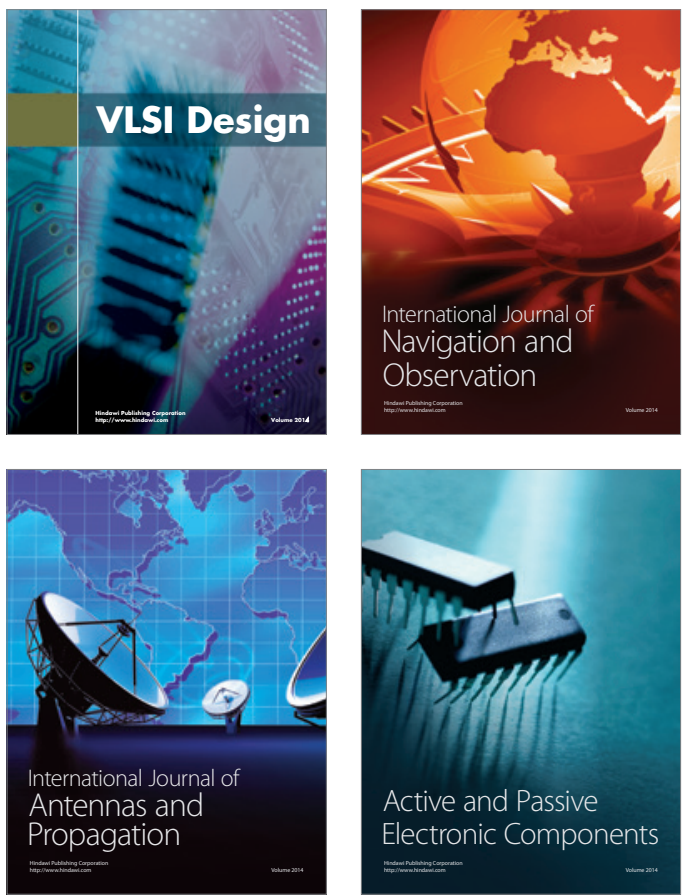
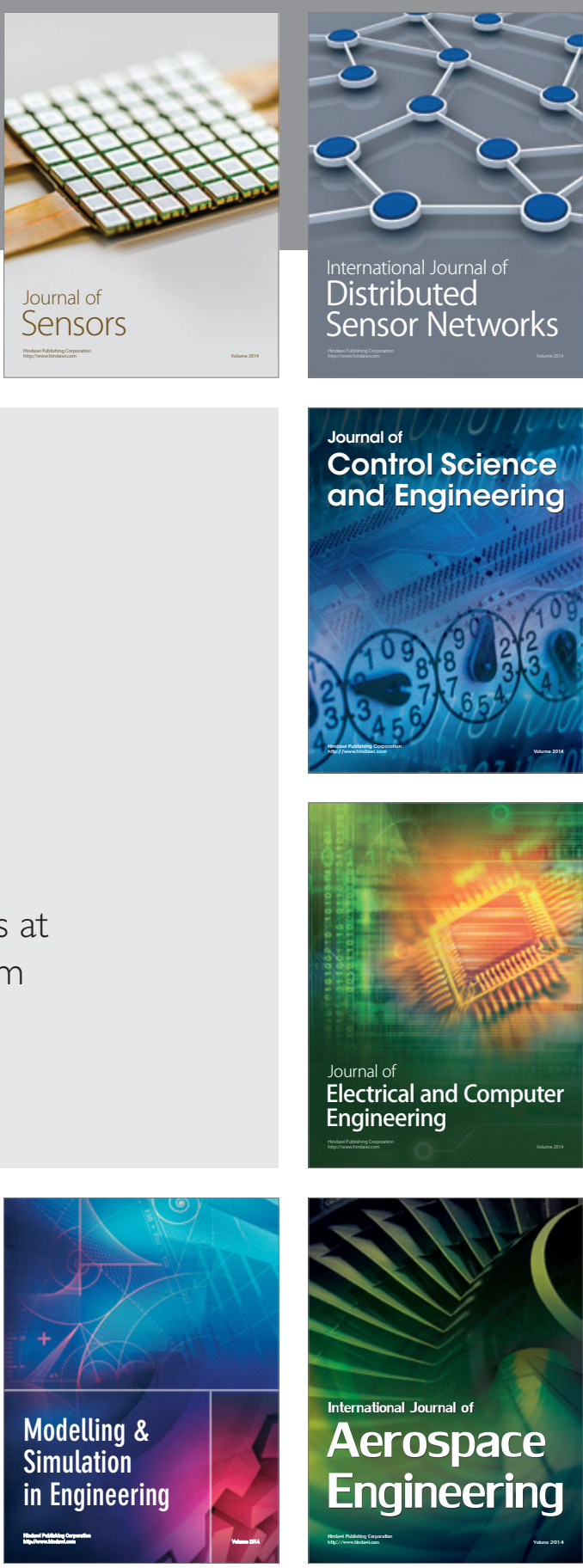

International Journal of

Distributed

Sensor Networks

Journal of

Control Science

and Engineering
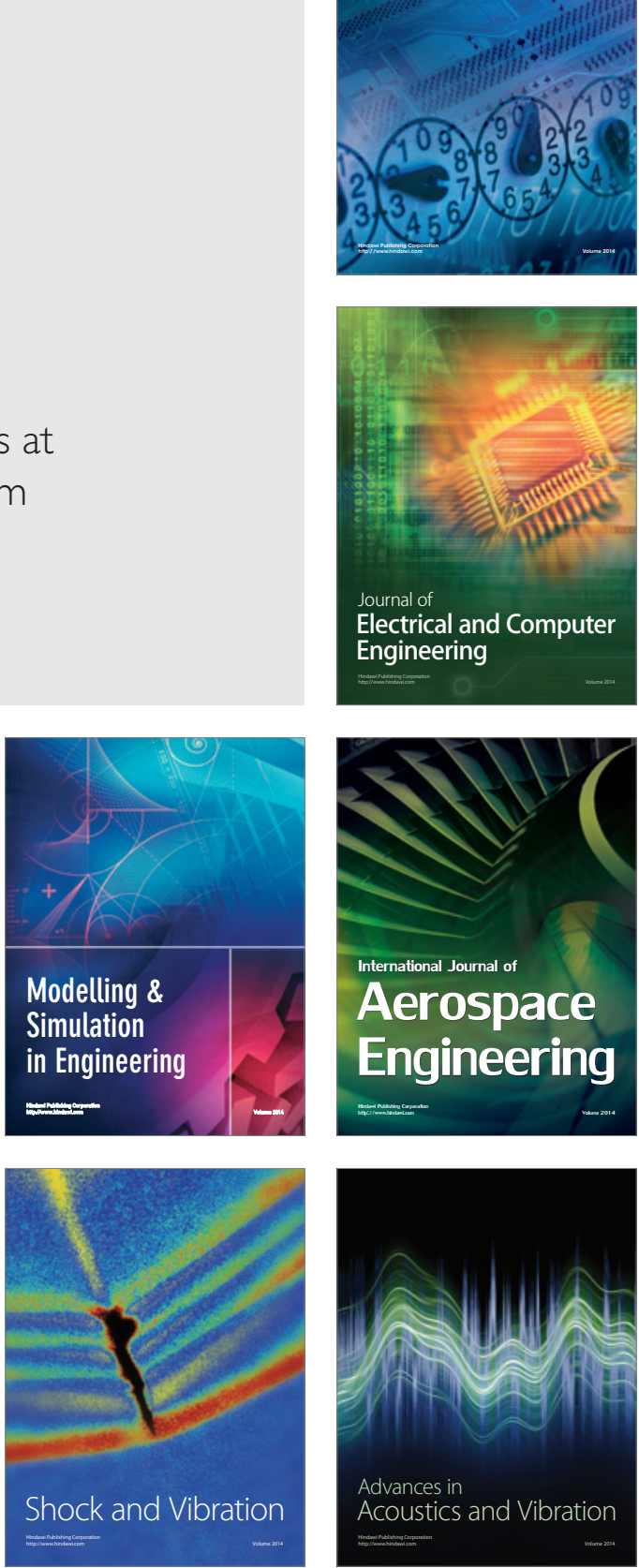